\title{
MiR-24 and miR-27 negatively regulate the expression of Th2 cells in children with idiopathic nephrotic syndrome
}

\author{
Cheng-rong $\mathrm{Li}^{1}$, Fen $\mathrm{Ni}^{1}$, Guang-lei Liu ${ }^{2}$, Jun Yang ${ }^{3}$, Shi-lei Jia ${ }^{4}$, Ran-ran Chen ${ }^{4}$, Li-bing \\ $\mathrm{Liu}^{3}$, and Xiao-jIe $\mathrm{Gao}^{1}$ \\ ${ }^{1}$ Shenzhen Children's Hospital \\ ${ }^{2}$ The Fifth Affiliated (Zhuhai) Hospital of Zunyi Medical University \\ ${ }^{3}$ Shenzhen Children's Hospital, Shenzhen \\ 4 Shenzhen Children's Hospital
}

May 5, 2020

\begin{abstract}
Purpose: This study was designed to investigate the effects of miR-24 and miR-27 on Th2 in children with non-atopic INS. Methods: Isolateing PBMCs by Ficoll density gradient, and transfected with human miR-24, miR-27 mimics/miR-24, miR-27 mimics control and miR-24, miR-27 inhibitors/miR-24, miR-27 inhibitor control. After that Real-time PCR to investigate the levels of microRNAs and IL-4mRNA, Flow cytometry to test the frequency of Th2 cells, and Cytometric bead array to measure the concentration of IgE, IL-4 and IL-13 in plasma. Results: The proportion of Th2 cells in peripheral blood of children with INS in the initial atopic and non-atopic groups were significantly higher $(\mathrm{P}<0.05)$, and there was no significant difference in the proportion of Th2 cells in the remission group $(\mathrm{P}>0.05)$. Plasma IgE, IL-4 and IL-13 were significantly increased in the initial atopic and non-atopic groups $(\mathrm{P}<0.05)$. MiR-24 and miR-27 were remarkably downregulated in the initial non-atopic group $(\mathrm{P}<0.05)$. The expressions of miR-24 and miR-27 were up-regulated in the initial non-atopic and control group, the proportion of Th2 cells and IL-4 mRNA expression were remarkably decreased $(\mathrm{P}<0.05)$, and the expressions of miR-24 and miR-27 were down-regulated, the proportion of Th2 cells and IL-4 mRNA expression were remarkably increased $(\mathrm{P}<0.05)$. Conclusion: There were high IgE in children with both atopic and non-atopic INS during the active period, which might be related to the high expression of IL-13 and IL-4 induced by the Th2 cells drifting. MiR-24 and miR-27 negative regulated the expressions of Th2 cells in INS.
\end{abstract}

\subsection{Introduction}

Idiopathic nephrotic syndrome(INS) is the most prevalent kidney disease in children, and is a main cause of chronic renal failure in children in China[1]. It is characterized by the clinical manifestation of proteinuria, low plasma albumin, hyperlipidemia, and edema. The prevalence is mostly preschool children, and the peak period is 3-5 years old. About 80-90\% of patients with kidney disease under 10 years of age are of minimal change disese (MCD)[2]. However, the specific etiology and mechanism of INS in children remains unclear. Previous studies have shown that the disease has obvious immune dysfunction, including humoral immune disorders, T cell subset dysfunction and abnormal secretion of cytokines, especially T cell dysfunction [3-8].

Th2 cells produce mainly IL-4 IL-5 and IL-13 and play an important role in parasitic infections and allergic inflammatory diseases[9-11]. As early as 1959, the association of pollen sensitivity and seasonal proteinuria has been reported, and about 30\% of children with INS have atopic manifestations such as allergic rhinitis and idiopathic dermatitis[12]. Some studies have also confirmed that IgE in INS is increased during active phase[13-15], however, it is still uncertain whether increased levels of IgE in childhood INS are pathogenetic or coincident. The first hit consists of the induction of CD80 in podocytes by microbial products, allergen, 
or T-cell cytokines such as IL-13 act on the glomeruli, resulting in the overexpression of podocyte CD80 and temporary proteinuria[16-18]. In normal settings, CD80 expression on podocytes is terminated by regulatory cytokines from Tregulatory cells and/or cytotoxic T-lymphocyte-associated and IL-10 by podocytes, and as a consequence, proteinuria is transient and mild[18-20]. However, we propose a second hit occurs in MCD and consists of abnormal censoring of podocyte CD80 expression due to a defective autoregulatory response by Tregs or by the podocyte itself. As a consequence, CD80 expression becomes persistent and nephrotic syndrome results [20]. Th2 related cytokines IL-13, a known stimulator of IgE response, may mediate proteinuria in patients with MCD because of its ability to directly induce CD80 expression on the podocyte[13-15, 18]. In recent years, studies have found that IL-13 and IL-4 were increased during the active phase of INS, IL-13 has involved in the pathogenesis of kidney disease[13-15, 18], but the mechanisms resulting in IL-13 increasing was still unclear. A few studies have also noticed that Th2 cells were overactivation in INS[8,18, 21-22], but the mechanisms resulting in Th2 cells over activation remained unknown, which needed to be studied to further explain the pathogenesis of INS immune.

MicroRNAs (miRNAs or miRs) are short non-coding RNAs of 20-23 nucleotides that regulate target gene expression in a post-transcriptional manner [23-24]. In brief, microRNAs can bind to the 3'-untranslated region (3'-UTR) of target mRNAs, leading to their translational inhibition or degradation [23-24]. Previously, miRNAs appeared more in the field of cancer and tumor research. Recently, miRNAs have also received much attention in kidney disease. Studies have shown that multiple miRNAs can inhibit the differentiation and function of Th2 cells[25-27]. In 2016, some scholars discovered that miR-24 and miR-27 act collaboratively through different downstream pathways to inhibit Th2 cell differentiation and IL-4 production [26-27]. However, it is unclear whether changes in miRNAs expression alter Th2 expression in active non-atopic INS, and its specific mechanism of action needs to be further study.

Therefore, in this study we systematically observes the dynamic changes of cytokines and miR-24, miR-27 involved in regulating Th2 cell differentiation in the active and remission phases, and observes the changes in the number and function of Th2 cells in children with non-atopic INS under different miRNAs expression. It may clarify the immune pathogenesis in INS, which may provide potential therapeutic targets and new ideas for the treatment of kidney disease.

\subsection{Materials and Methods}

\subsection{Subjects}

Fouty children with INS (28 males and 12 females; median age: 38 months; age range: 22-55 months) were enrolled in this study. The study participants were recruited from the inpatient pediatric population at Shenzhen Children's Hospital between the dates of September 2015 and October 2016. These children met all the inclusion criteria: 1) The diagnostic criteria for INS prepared by 2010 evidence-based diagnosis and treatment guidelines for common kidney diseases in pediatrics in China ; 2) All of them were hormonesensitive children, that is, children with negative urine protein within 4 weeks of treatment with sufficient hormones and no other immunosuppressants; 3) No secondary kidney disease (such as secondary nephrotic syndrome, nephrotic nephrotic syndrome or congenital kidney disease etc.); 4) No other systemic viscera syndrome; 5) Signed informed consent. According to the condition, they were divided into first-onset group (20 cases, atopic constitution, 6 cases, 14 cases of non-atopic constitution) and remission group (20 cases, atopic constitution, 6 cases, non-atopic constitution 14 cases). Defined as children with nephrotic syndrome for the first time, the selection criteria for remission patients were children with kidney disease who had stopped hormones for more than 4 weeks and had normal blood biochemical and urine tests. Tweety agesimilar matched children (11 males and 9 females; median age: 31.3 months; age range: 18-112 months) who came to our hospital for physical examination during the same period were enrolled in the healthy controls (Ctrl). Informed consent was obtained from the parents of all patients as a condition of study enrollment, and the study was carried out with pre-approval by the local Medical Ethics Committee.

\subsection{Blood samples}

Venous blood was collected from each of the PNS patients and each of the healthy controls and stored 
in ethylenediamine tetraacetic acid (EDTA)- $\mathrm{Na}_{2}$ as an anti-coagulant. Peripheral blood mononuclear cells (PBMCs) were isolated by Ficoll density gradient for subsequent flow cytometric analysis . Plasma was obtained after centrifugation and stored at $-80^{\circ} \mathrm{C}$ for analysis by cytometric bead array (CBA). CD4 $4^{+} \mathrm{CD} 25^{-} \mathrm{T}$ cells were immediately isolated from the patients' peripheral blood samples, according to the manufacturer's instructions for microbeads (11363D, Dynal; Invitrogen, USA). Cell populations were considered pure at > $97 \%$ by flow cytometry, and cell activity was investigated using the trypan blue exclusion assay (using the threshold of $>95 \%$ of the cell population as a positive indicator of significantly decreased cell activity).

\subsection{Total RNA extraction and cDNA synthesis}

Total RNA (including miRNAs) was isolated from $\mathrm{CD} 4^{+} \mathrm{CD} 25^{-} \mathrm{T}$ cells according to the manufacturer's instructions using the miRNeasy Mini Kit (Qiagen, Germany). The integrity of the isolated total RNA was confirmed by an average $\mathrm{OD}_{260} / \mathrm{OD}_{280} \mathrm{~nm}$ absorption ratio of 1.98. cDNA was synthesized with oligo-dT primers and RevertAid ${ }^{\mathrm{TM}} \mathrm{H}$ Minus reverse transcriptase (Fermentas, Lithuania). The miRNA cDNA was synthesized with the miScript II RT Kit from Qiagen. Negative control samples (no first-strand synthesis) were prepared by performing reverse transcription in the absence of reverse transcriptase.

\subsection{LightCycler real-time PCR}

The cDNA levels of IL-4 was quantitated by real-time PCR using the Quantitect SYBR green PCR Kit (Takara, Japan) and a LightCycler(r) 2.0 (Roche Molecular Biochemicals, Switzerland). The primers of IL-4 used for RT-PCR were as follows: 5'-TCATTTTCCCTCGGTTTCAG-3'(forward) and 5'ATAGGTGTCGATTTGCAGTG-3' (reverse). The cDNA levels of miR-24 and miR-27 were quantitated by real-time PCR using the miScript SYBR Green PCR Kit (Qiagen) and the LightCycler(r) 2.0. The primers used for miRNA real-time PCR were designed according to the recommendations for use with the miRNA miScript Primer Assay (Qiagen). The second derivative maximum method was performed for CP determination using LightCycler software V3.5.30 (Roche Molecular Biochemicals). After normalization with Relative Quantification Software V1.0 (Roche Molecular Biochemicals), the final results were calculated as ratios of the target genes or miRNAs to the amount of GAPDH or U6.

\subsection{Flow cytometry analysis of Th2 cells}

To measure cytoplasmic expression of markers by Th2 cells, cells cultured in a $37 * \mathrm{C} / 5 \% \mathrm{CO} 2$ incubator were stimulated for $24 \mathrm{~h}$ with ionomycin (250 ng/ml; SigmaAldrich), PMA (25 ng/ml; Sigma-Aldrich), and monensin (20 ng/mL; eBio-science, San Diego, CA, USA). The cells were then stained for surface markers,

fixed, and permeabilized using the Cytofix/Cytoperm kit (eBioscience), and then stained with antibodies specific for intracellular cytokines or with corresponding isotype-matched controls, followed by analysis using a FACS Canto II flow cytometer (BD Biosciences, Mississauga, ON, Canada). The vitality of cells was discriminated using fixable viability stain 450 (FVS450, BD Biosciences). Validated antibodies (CD3eFour450A 、 CD4-FITC、IL-4-PE) were purchased from eBioscience.

\subsection{CBA detection of plasma IgE, IL-4 and IL-13}

The plasma levels of IgE, IL-4 and IL-13 were measured using a CBA kit (eBioscience) according to the manufacturer's instructions. All samples were measured in duplicate.

\subsection{Cell transfection and culture}

To investigate the role of miR-24 and miR-27 in Th2 cells, we performed miR-24 and miR-27 transfection experiments in PBMCs. PBMCs were isolated by Ficoll density gradient. After isolation, Ctrl-m, miR-24-m, or miR-27-m, Ctrl-i, miR-24-i, or miR-27-i (obtained from RiboBio; Guangzhou, China) were transfected into the PBMCs using a riboFECT CP Transfection Kit (Guangzhou, China). Cells were cultured in a 24-well plate at $3 \times 10^{6}$ cells per ml of RPMI-1640 medium (Gibco, CA, USA) supplemented with $15 \%$ fetal calf serum (Gibco, CA, USA) and maintained at 37 with $5 \%$ CO2. Finally, cells were harvested for flow cytometry and RT-PCR analysis. 


\subsection{Statistical analysis}

SPSS software for Windows version 13.0 was used for statistical analysis (SPSS Inc., USA). The data are expressed as mean \pm standard deviation. One-way analysis of variance was used to compare multiple groups, and Student's $t$ - test was used to compare 2 groups. $P$-values $<0.05$ were considered indicative of statistical significance.

\subsection{Results}

\subsection{Atopic and non-atopic INS have increased serum IgE levels.}

We detected plasma levels of IgE by CBA (Fig. 1). Compared with the control group, plasma IgE concentrations in the initial atopic group (1350.67 \pm 837.39 vs. $57.76 \pm 48.25, \mathrm{P}<0.05)$ and the initial non-atopic group $(441.01+-357.45$ vs. $57.76+-48.25, \mathrm{P}<0.05)$ were significantly increased, there was no significant difference between remission atopy group and remission non-atopic group $(237.21+-121.58$ vs. $60.86+-$ $57.99, P>0.05$ ); Compared with the initial atopic group, the other groups were significantly decreased; Compared with the initial non-atopic group, the initial atopic group was significantly higher, the control and the remission non-atopic group were significantly reduced, and there was no significant difference in the remission atopic group.

3.2 Active phase INS patients Th2 cells were over-activation and the expression of associated factors were increased.

The number of Tregs from patients' whole blood was quantified by flow cytometry (Fig. 2). The proportion of peripheral Th2 cells in initial atopic group $(5.60+-1.21$ vs. $3.29+-1.02, \mathrm{P}<0.05)$ and the initial non-atopic group $(4.23+-0.92$ vs. $3.29+-1.02, \mathrm{P}<0.05)$ were significantly increased than that of healthy subjects, there was no significant difference between remission atopy group and remission non-atopic group $(3.60+-0.85$ vs. $3.33+-0.95, P>0.05)$; Compared with the initial atopic group, the other groups were significantly decreased; Compared with the initial non-atopic group, the initial atopic group was significantly higher, the control and the remission non-atopic group were significantly reduced, and there was no significant difference in the remission atopic group (Fig. 2A, B). We detected plasma levels of IL-4 and IL-13 by CBA (Fig. 2C, D) and found that plasma IL-4 and IL-13 concentrations were significantly increased in initial atopic group (IL-4: $66.66+-43.85$ vs. $23.29+-13.91, P<0.05$; IL-13: $65.15+-16.50$ vs. $15.66+-8.16, P<0.05$ ) and the initial non-atopic group (IL-4: $37.04+-17.39$ vs. $23.29+-13.91, P<0.05$; IL-13: $33.15+-14.09$ vs. 15.66+-8.16, $P<0.05)$, there was no significant difference between remission atopy group and remission non-atopic group $(22.09+-10.32$ vs. $16.47+-11.69, P>0.05)$; Compared with the initial atopic group, the other groups were significantly decreased; Compared with the initial non-atopic group, the initial atopic group was significantly higher, the control and the remission non-atopic group were significantly reduced, and there was no significant difference in the remission atopic group.

\subsection{The expression levels of miR-24 and miR-27 in non-atopic INS patients.}

It has previously been shown that miR-24 and miR-27 have important effects on Th2 cells expression. Because we saw decreased expression of Th2 cells in active non-atopic INS, we wanted to determine whether the associated miRNAs were also decreased. As shown in Figure 3, the levels of miR-24 and miR-27 were significantly downregulated compared to controls during the active phase of non-atopic INS (miR-24: $(21.84+-6.86$ vs. 46.03+-10.08) $\times 10^{-3}, P<0.05$; miR-27: (20.72+-4.93 vs. $\left.\left.37.83+-6.83\right) \times 10^{-3}, P<0.05\right)$. MiR-24 and miR-27 were remarkably upregulated in the remission group , but not up to control levels (miR-24: (35.59+-9.10) x10-3, $P<0.05 ;$ miR-27: $\left.(32.55+-7.86) \times 10^{-3}, P<0.05\right)$.

3.4 MiR-24 and miR-27 negative regulated the expressions of Th2 cells.

To further determine the relationship between miR-24 - miR-27 and Th2 cells, we transfected PBMCs isolated from healthy volunteers and active non-atopic INS with with a miRNA negative control (Ctrl-m), a miR-24/miR-27 mimic (miR-24-m, miR-27-m), a miRNA inhibitor negative control (Ctrl-i), or a miR24/miR-27 inhibitor (miR-24-i, miR-27-i). Up regulating the expression of miR-24(Fig. 4A; Fig. 4B;) and 
miR-27(Fig. 5A; Fig. 5B), the frequency of Th2 cells(Fig. 4C, D; Fig. 5C,D) and the expression of IL-4 mRNA(Fig. 4E, F; Fig. 5E, F) were all down regulated. On the contrary, down regulating the expression of miR-24(Fig. 4G; Fig. 4H) and miR-27(Fig. 5G; Fig. 5H), the frequency of Th2 cells(Fig. 4I, J; Fig. 5I, $\mathrm{J}$ ) and the expressions of IL-4 mRNA(Fig. 4K, L; Fig. 5K, L) were all up regulated.

\subsection{Discussion}

A large body of evidence has demonstrated that the immune system may play a crucial role in INS[3]. To date, the underlying pathogenesis of INS remains largely unknown. Some researchers hypothesize that INS may be caused by dysregulation of $\mathrm{T}$ lymphocytes or an abnormal $\mathrm{T}$ cell response[3-8]. MCD is the most common cause of INS[1-2,18-19]. Th2 cells play an important role in allergic inflammatory diseases[9$11,25,28]$. The association of pollen sensitivity and seasonal proteinuria was first reported more than 60 years ago[12]. Minimal change nephrotic syndrome is often associated with clinical features of allergy such as bronchial asthma, allergic rhinitis, atopic dermatitis and urticaria. Some studies have also confirmed that patients with INS also may show increased serum IgE levels, especially in children with repeated recurrence[13-15,18]. However, the causal relationship between the increase of serum $\operatorname{IgE}$ or atopy and the pathogenesis of INS remains to be further explored. This study found that the plasma IgE during the active phase was significantly increased in the initial atopic and non-atopic groups compared with the control group, and it also significantly decreased during the remission phase. In remission, the IgE level of non-atopic INS children tended to be normal, while IgE level of atopic was still high. These results suggested that atopic and non-atopic INS have increased serum IgE levels, but further investigation of the cytokine regulating network of serum IgE was necessary to clarify the relationship between IgE production and INS.

IL-13 and IL-4 secreted by Th2 cells can promote the production of IgE in B cells[9-11]. IL-13 is known to induce a switch from IgM to IgE in B cells and induce CD80 expression by podocytes. Concomitantly, increased CD80 expression by podocytes is associated with proteinuria[13-15, 18]. And, IL-13 is present in urine and serum of patients with kidney disease and is associated with a large amount of proteinuria[13$15,18]$, which suggesting that IL-13 is related to the pathogenesis of kidney disease, but the mechanisms resulting in IL-13 increasing was still unclear. In recent years, studies have found that Th2-related factors IL-13 and IL-4 are increased in active INS [13-15, 18]. A few studies have also noted that the proportion of Th2 cells in INS is increased[8,18, 21-22], but It is still controversial whether the proportion is abnormally increased, so this article further tested the expression of Th2 cells in peripheral blood and the concentrations of IL-13 and IL-4 in the plasma. our data demonstrate that the expression levels of Th2-related factors ( IL-13, IL-4 ) was significantly increased in the initial atopic and non-atopic groups, at the same time, Th2 cells were over-activation. These results suggested that the proteinuria and increased IgE levels in the initial atopic and non-atopic during the active phase might be related to the high expression of IL-13 and IL-4 which were caused by Th2 drifting, but the mechanism that lead to Th2 drifting still needed to be studied to further explain the pathogenesis of INS immune.

MiRNAs are subtle master controllers of gene expression and therefore play a crucial role in the pathogenesis of human diseases, especially in chronic multifactorial diseases[23-24]. Some specific miRNAs have been reported to be abnormally expressed in various allergic and autoimmune diseases such as asthma, SLE, Lupus Nephritis and are associated with disease activity [25, 29-30]. MiRNAs are important for Th2 cell proliferation, differentiation and immune function[25-27]. MiR-24 and 27 suppress allergic inflammation and target a network of regulators of Th2 cell-associated cytokine production[26-27]. However, it is unclear whether changes in miRNAs expression alter Th2 expression in active INS. Therefore, In this study we further observed the expression changes of miR-24 and miR-27 involved in regulating Th2 cell differentiation. The results showed that miR-24 and miR-27 were lowly expressed in children with active non-atopic INS, suggesting that Th2 cells drifting might be related to the low- expression of miR- 24 and miR-27.

In order to verify whether this hypothesis was true, we used cell transfection technology to transfer miRNA24 or miR-27 mimic to non-atopic and healthy control group mononuclear cells to increase the expression level, and transfer miR-24 or miR-27 inhibitor to reduce the expression level. The normal control group was also set up to verify the effect of transfection. The results showed that the proportion of Th2 cells and 
the expression of Th2 cell-associated cytokine IL- 4 were decreased in the miR-24 and miR-27 up-regulated groups. In contrast, the proportion of Th2 cells and the expression of Th2 cell-associated cytokine IL- 4 were increased in miR-24 and miR-27 down-regulated groups, indicating that miR-24, miR- 27 negatively regulated Th2 cell expression. Combining the expression trends of miR-24, miR-27 and Th2 cells obtained from patients' peripheral blood mononuclear cells, our hypothesis was true, miR-24 and miR-27 negatively regulated the change in the proportion of Th2 cells, and they might play an important role in Th2 expression in active period INS.

In conclusion, Th2 related-cytokine IL-13 was related to the pathogenesis of kidney disease, but the mechanisms resulting in IL-13 increasing was still unclear. Our results suggested that there were high $\operatorname{IgE}$ in children with both atopic and non-atopic INS during the active period, which might be related to the high expression of IL-13 and IL- 4 induced by Th2 cells drifting. The expressions of miR-24 and miR-27 were down-regulated in the initial non-atopic INS, the proportion of Th2 cells and IL-4mRNA expression were remarkably increased, which leading to IL-13 over-expression and increased IgE levels. That was, miR-24 and miR-27 negative regulated the expressions of Th2 cells and played an important role in the change of Th2 expression in children with active INS. Therefore, we speculated that miRNAs would be potential therapeutic targets for kidney disease, our research has provided a new research direction and treatment method for the treatment of INS.

\section{Acknowledgments}

This study was supported by grants from the Shenzhen Municipal Government of China (no. JCYJ20160429174400950); the Sanming Project of Medicine in Shenzhen (no. SZSM201812002). The authors are also grateful to the patients and healthy volunteers who participated in this study.

\section{Disclosure Statement}

The authors have no conflicts of interest to declare.

\section{References}

1. Yang JY, Yao Y. Analysis of 1268 patients with chronic renal failure in childhood: a report from 91 hospitals in China from 1990 to 2002. Zhonghua Er Ke Za Zhi Chinese Journal of Pediatrics. 2004;42:72430.

2. Vivarelli M, Massella L, Ruggiero B, et al. Minimal Change Disease[J]. Clinical Journal of the American Society of Nephrology, 2016, 12(2):332.

3. Colucci M, Corpetti G, Emma F, et al. Immunology of idiopathic nephrotic syndrome[J]. Pediatric Nephrology, 2017:1-12.

4. Sellier-Leclerc A-L, Duval A, Riveron S et al. A humanized mouse model of idiopathic nephrotic syndrome suggests a pathogenic role for immature cells. J Am Soc Nephrol. 2007;18:2732-9.

5. Wang L, Li Q, Wang L et al. The Role of Th17/IL-17 in the Pathogenesis of Primary Nephrotic Syndrome in Children. Kidney Blood Press Res. 2013;37:332-45.

6. Fodor P, Saitúa MT, Rodriguez E, González B, Schlesinger L. T-Cell Dysfunction in Minimal-Change Nephrotic Syndrome of Childhood. Am J Dis Child. 1982;136:713-7.

7. Herrod HG, Stapleton FB, Trouy RL, Roy S. Evaluation of T lymphocyte subpopulations in children with nephrotic syndrome. Clin Exp Immunol. 1983;52:581-5.

8. Kanai T, Shiraishi H, Yamagata T et al. Th2 cells predominate in idiopathic steroid-sensitive nephrotic syndrome. Clin Exp Nephrol. 2010;14:578.

9. Forsthuber T G . Stability of T-cell lineages in autoimmune diseases[J]. Expert Review of Clinical Immunology, 2012, 8(4):299-301. 10. Tosolini M, Kirilovsky A, Mlecnik B, et al. Clinical Impact of Different Classes of Infiltrating T Cytotoxic and Helper Cells (Th1, Th2, Treg, Th17) in Patients with Colorectal Cancer[J]. 
Cancer Research, 2011, 71(4):1263-71. 11. Chapoval S, Dasgupta P, Dorsey N J, et al. Regulation of the T helper cell type 2 (Th2)/T regulatory cell (Treg) balance by IL-4 and STAT6[J]. Journal of Leukocyte Biology, 2010, 87(87):1011-1018. 12. Hardwicke J, Soothill JF, Squire JR, Holti G. Nephrotic syndrome and pollen hypersensitivity. Lancet 1959; I: 499-502. 13. Mishra O P, Teli A S, Singh U, et al. Serum Immunoglobulin E and Interleukin-13 Levels in Children with Idiopathic Nephrotic Syndrome[J]. Journal of Tropical Pediatrics, 2014, 60(6): 467-471. 14. Hossain A. Serum IgE level in relapsing idiopathic nephrotic syndrome in children[J]. Urology \& Nephrology Open Access Journal, 2019, 7(2).

15. Mishra, O. P . Serum Immunoglobulin E in Idiopathic Nephrotic Syndrome[J]. Journal of Tropical Pediatrics, 2004, 50(3):149-152.

16. Garin E H , Diaz L N, Mu W, et al. Urinary CD80 Excretion Increases in Idiopathic Minimal-Change Disease[J]. Journal of the American Society of Nephrology, 2009, 20(2):260-266.3.

17. Reiser, J. Danger Signaling by Glomerular Podocytes Defines a Novel Function of Inducible B7-1 in the Pathogenesis of Nephrotic Syndrome[J]. Journal of the American Society of Nephrology, 2004, 15(9):22462248 .

18. Abdel-Hafez M, Shimada M , Lee P Y , et al. Idiopathic Nephrotic Syndrome and Atopy: Is There a Common Link?[J]. American Journal of Kidney Diseases, 2009, 54(5):945-953.

19. Shimada, M., Araya, C., Rivard, C. et al. Minimal change disease: a "two-hit" podocyte immune disorder?[J]. Pediatr Nephrol, 2011, 26(4): 645-649.

20. Carlos, Araya, Leila, et al. T regulatory cell function in idiopathic minimal lesion nephrotic syndrome[J]. Pediatric Nephrology, 2009.

21. Adrogue H E , Borillo J , Torres L, et al. Coincident Activation of Th2 T Cells with Onset of the Disease and Differential Expression of GRO-Gamma in Peripheral Blood Leukocytes in Minimal Change Disease[J]. American Journal of Nephrology, 2007, 27(3):253-261.

22. Carlos E. Araya, Clive H. et al. A case of unfulfilled expectations. Cytokines in idiopathic minimal lesion nephrotic syndrome[J]. Pediatric Nephrology, 21(5):603-610.

23. Dai R, Ahmed S A. MicroRNA, a new paradigm for understanding immunoregulation, inflammation, and autoimmune diseases[J]. Translational Research, 2011, 157(4): 163-179. 24. Pauley K M, Cha S, Chan E K L. MicroRNA in autoimmunity and autoimmune diseases[J]. Journal of autoimmunity, 2009, 32(3): 189-194.. 25. Simpson L J, Patel S, Bhakta N R, et al. A microRNA upregulated in asthma airway T cells promotes TH2 cytokine production[J]. Nature Immunology, 2014, 15(12):1162-70. 26. Pua H H, Steiner D F, Patel S, et al. MicroRNAs 24 and 27 suppress allergic inflammation and target a network of regulators of T helper-2 cell-associated cytokine production[J]. Immunity, 2016, 44(4):821-832. 27. Sunglim Cho, ChengJang $\mathrm{Wu}$, Tomoharu Yasuda, et al. miR-23 2724 clusters control effector T cell differentiation and function[J]. Journal of Experimental Medicine, 2016, 213(2):jem.20150990.

28. NakayamaToshinori, HiraharaKiyoshi, OnoderaAtsushi, et al. Th2 Cells in Health and Disease[J]. Annual Review of Immunology, 2017, 35(1):53-84.

29. Mohsen H , Paulina K, Ekaterina V R , et al. The Involvement of MicroRNAs in Modulation of Innate and Adaptive Immunity in Systemic Lupus Erythematosus and Lupus Nephritis[J]. Journal of Immunology Research, 2018, 2018:1-15.

30. Stypińska Barbara, Agnieszka P G . Cytokines and MicroRNAs as Candidate Biomarkers for Systemic Lupus Erythematosus[J]. International Journal of Molecular Sciences, 2015, 16(10):24194-24218.

\section{Figure Legends}

Fig. 1. Atopic and non-atopic INS have increased serum IgE levels. Fouty children with INS and tweety control subjects were enrolled in this study. Fourteen patients were non-atopic constitution, six 
patients were atopic constitution. Data are shown as mean $\pm \mathrm{SD} .{ }^{*} P<0.05 .{ }^{* *} P>0.05$. Ctrl, healthy control; AA, acute allergy; ANA, acute non-allergy; RA, remission atopy; RNA, remission non-allergy.

Fig. 2. The expression of Th2 cells and Th2-related factors from patients with INS. A. Flow cytometry histogram for Th2 cells.B. The proportions of Th2 cells. C. Plasma concentrations of IL-4. D. Plasma concentrations of IL-13. Data are shown as mean \pm SD. Fouty children with INS and tweety control subjects were enrolled in this study. Fourteen patients were non-atopic constitution, six patients were atopic constitution. Data are shown as mean $\pm \mathrm{SD} .{ }^{*} P<0.05$. ${ }^{* *} P>0.05$. Ctrl, healthy control; AA, acute allergy; ANA, acute non-allergy; RA, remission atopy; RNA, remission non-allergy.

Fig. 3. The expression of miRNAs in $\mathrm{CD}^{+}{ }^{+} \mathrm{CD25}^{-} \mathrm{T}$ cells from INS patients. Relative expression levels of miRNAs were determined by real-time PCR using U6 as an endogenous reference. Normalized values are derived from the ratios of target miR/U6 expression, shown as mean $\pm \mathrm{SD}$. Fourteen patients with nonatopic constitution INS and tweety control subjects were enrolled in this study. Data are shown as mean \pm SD. ${ }^{*} P<0.05 .{ }^{* *} P>0.05$. Ctrl, healthy control; ANA, acute non-allergy; RNA, remission non-allergy.

Fig.4. and Fig.5. MiR-24 and miR-27 negative regulated the expressions of Th2 cells. Relative expression levels of miRNAs or IL- 4 were determined by real-time PCR using U6 or GAPDH as an endogenous reference gene. Normalized values are derived from the ratios of target miR/U6 or gene/GAPDH mRNA expression, shown as mean \pm SD. Six children with active non-atopic INS and six control subjects were enrolled in this study. Data are shown as mean $\pm \mathrm{SD} .{ }^{*} P<0.05 .{ }^{* *} P>0.05$. Ctrl, healthy control; INS, idiopathic nephrotic syndrome; Ctrl-m, miRNA negative control; miR-24-m/miR-27-m, miR-24/miR27 mimic; Ctrl-i, miRNA inhibitor negative control; miR-24-i/miR-27-i, miR-24/miR-27 inhibitor.

\section{Fig.1}

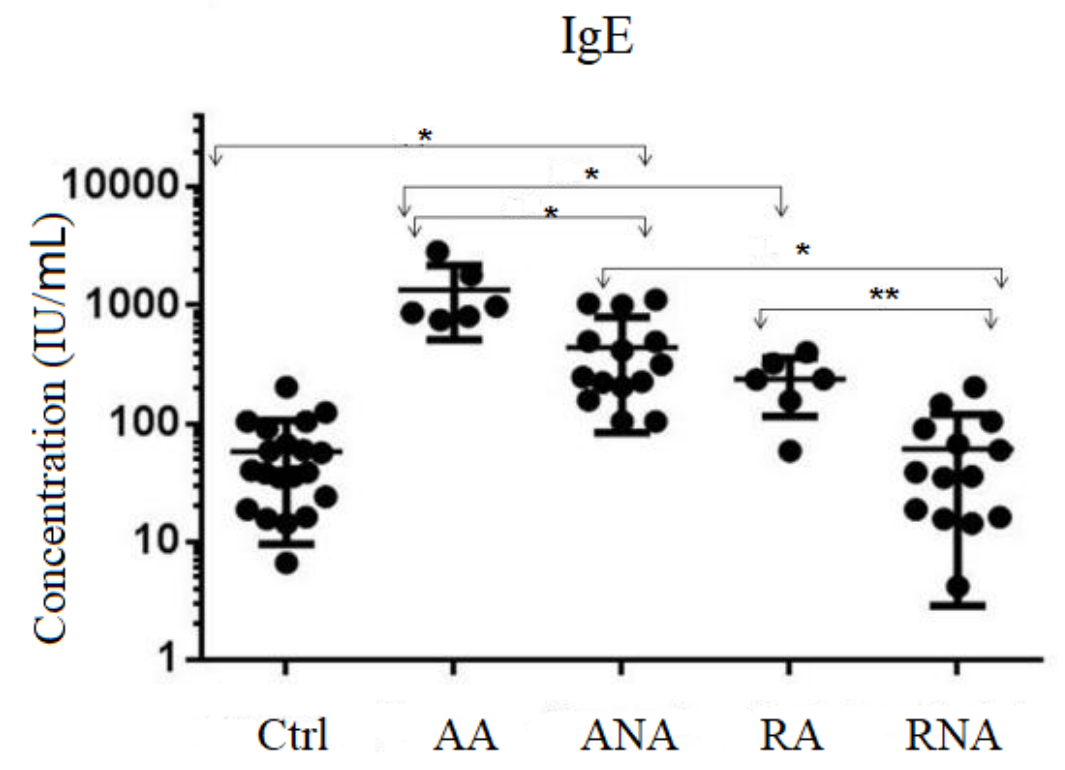



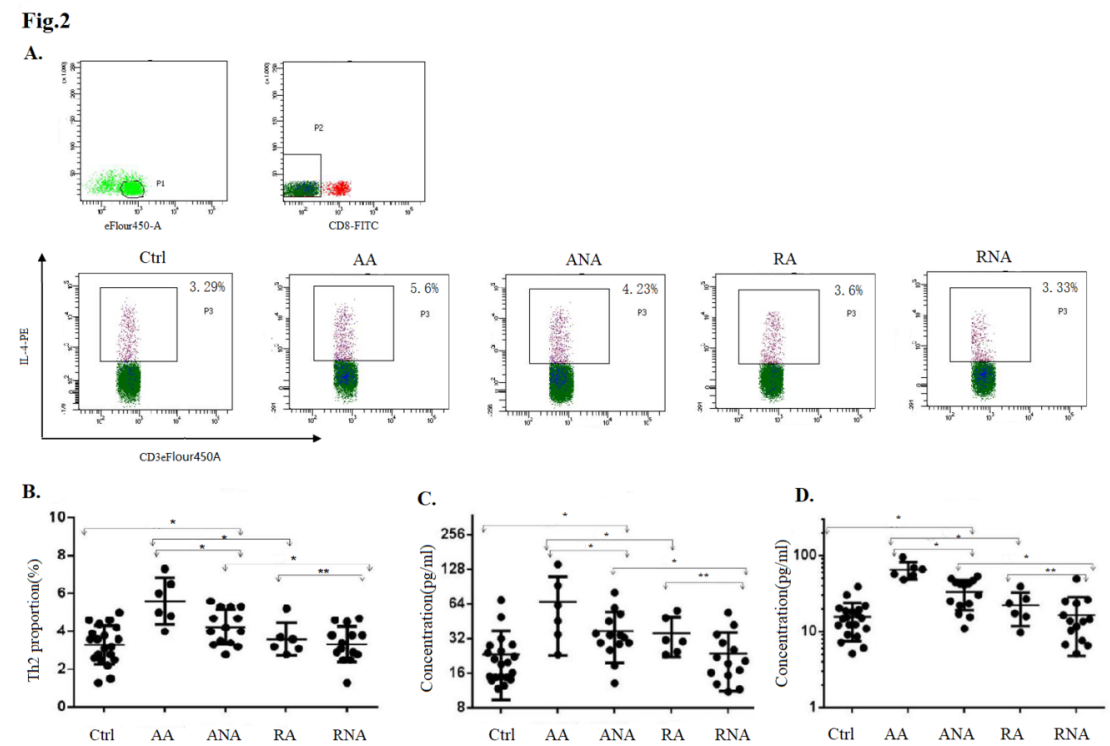

Fig.3

A.

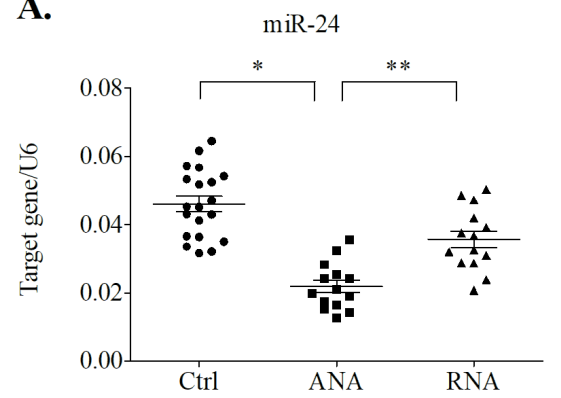

B.

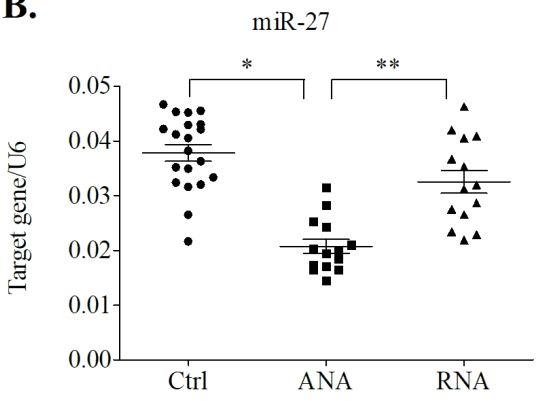


Fig.4

A.

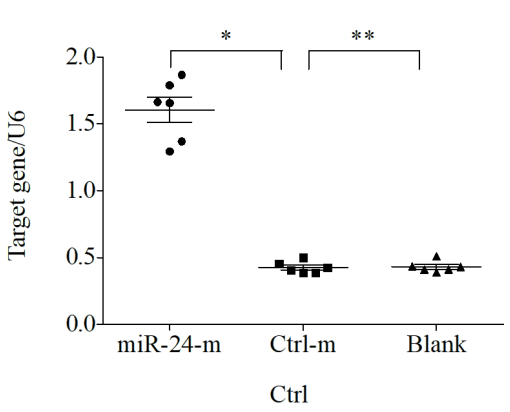

C.

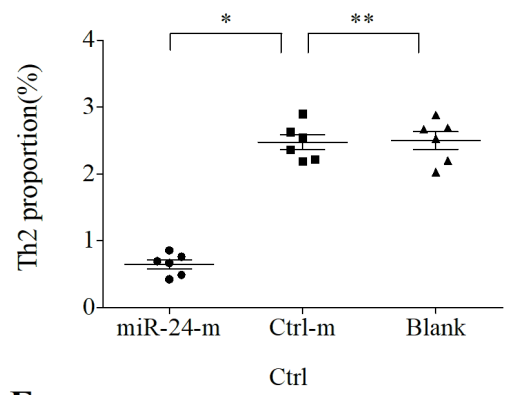

E .

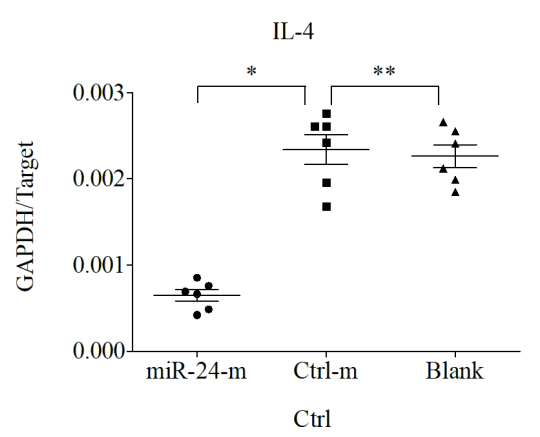

B .

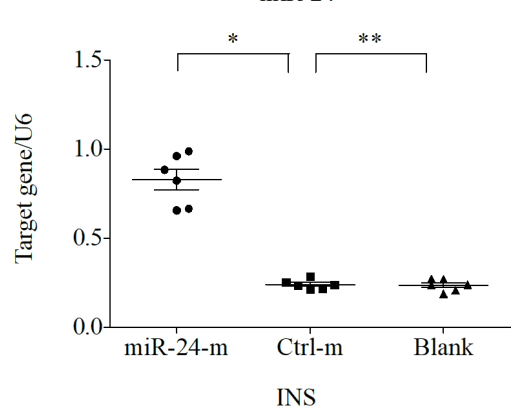

D.

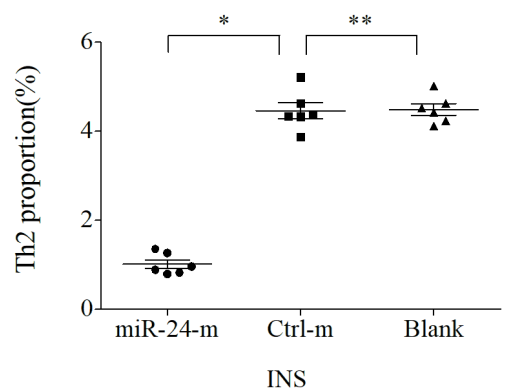

F .

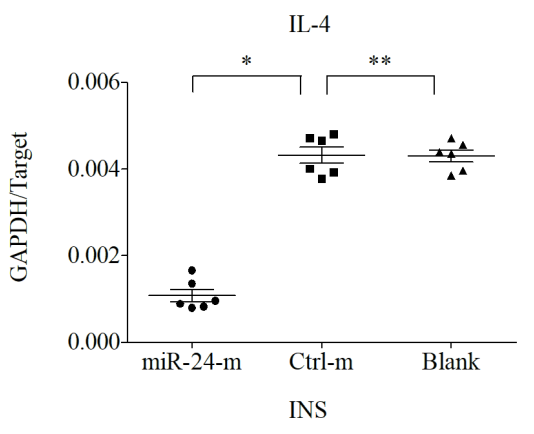


G.

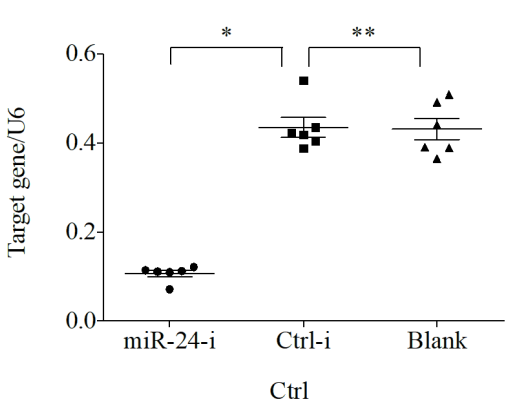

I .

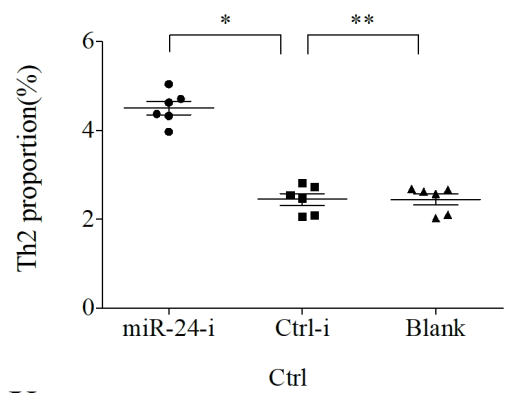

K.

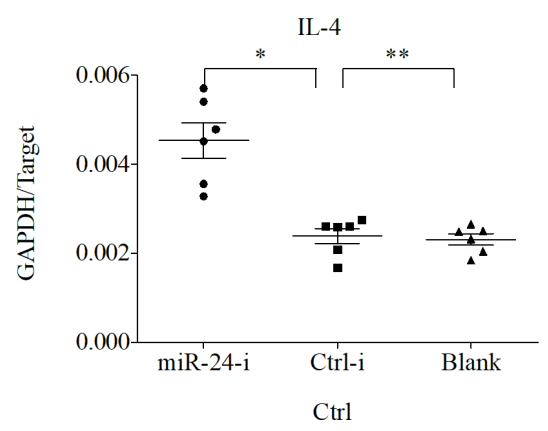

H.

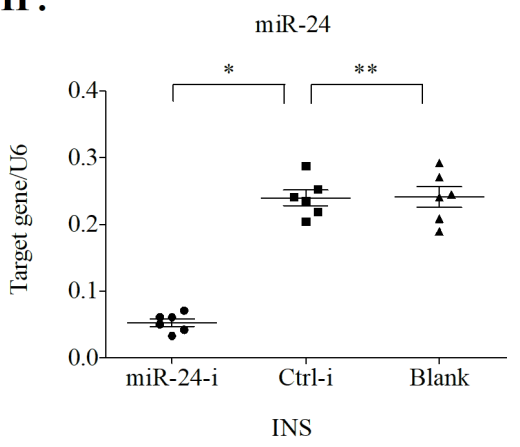

J.

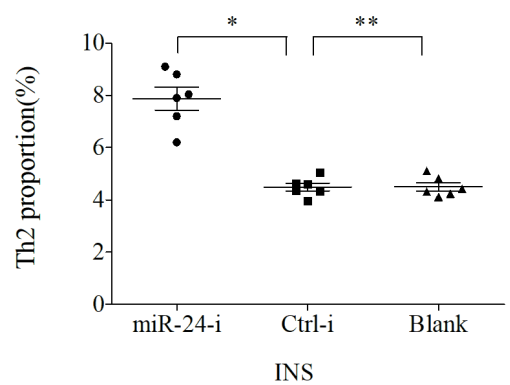

L .

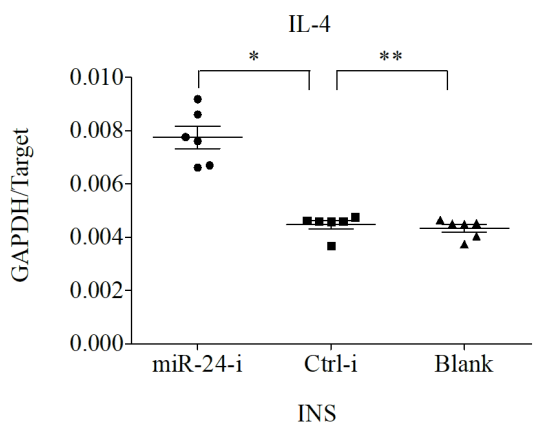


Fig.5

A.

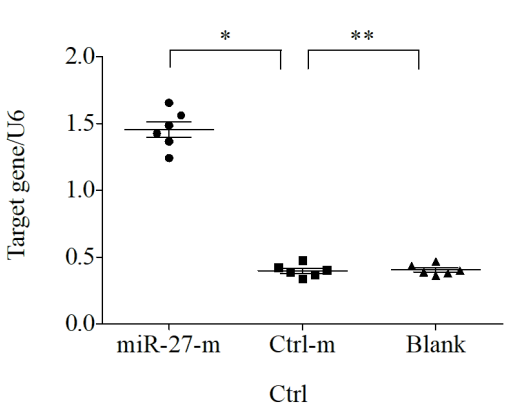

C.

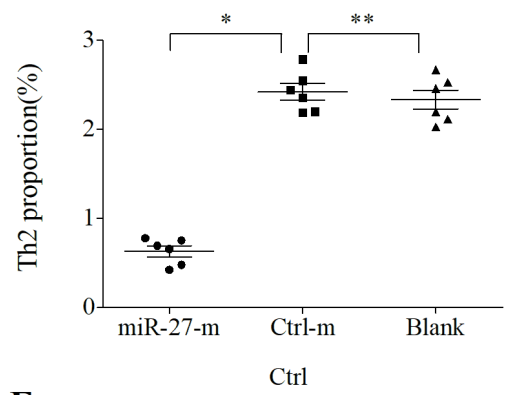

E .

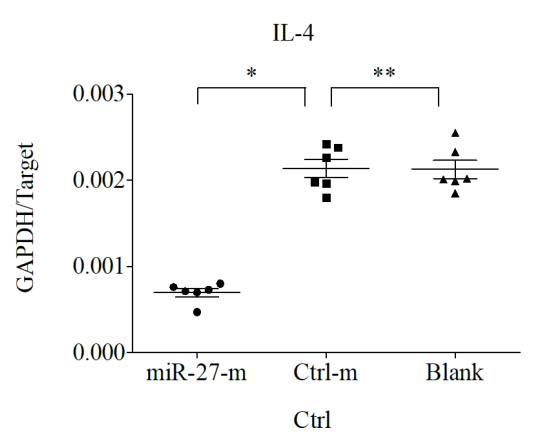

B .

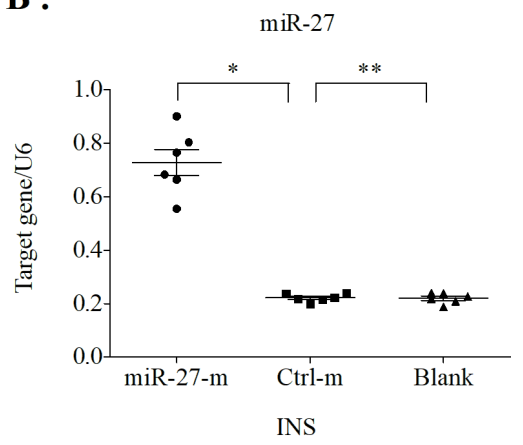

D.

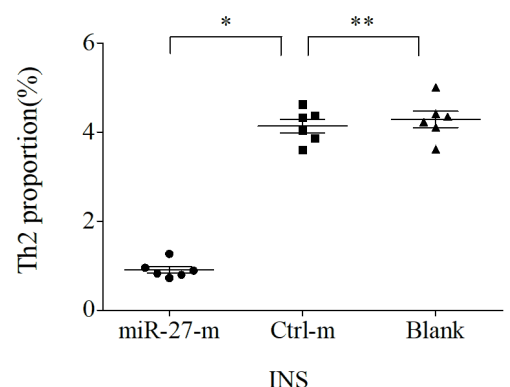

F .

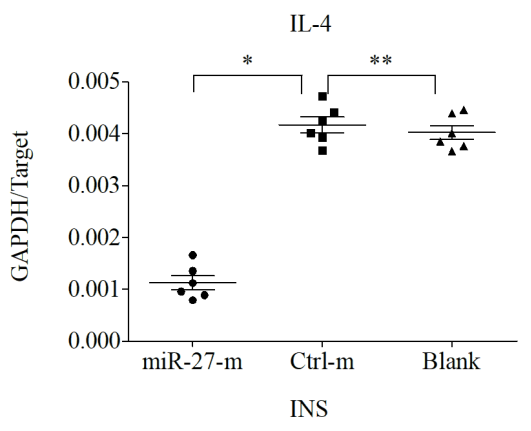


G.

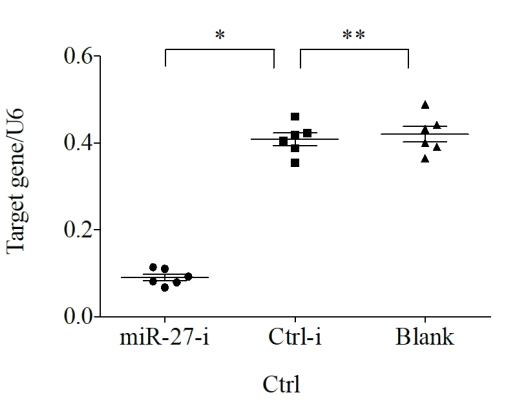

I .

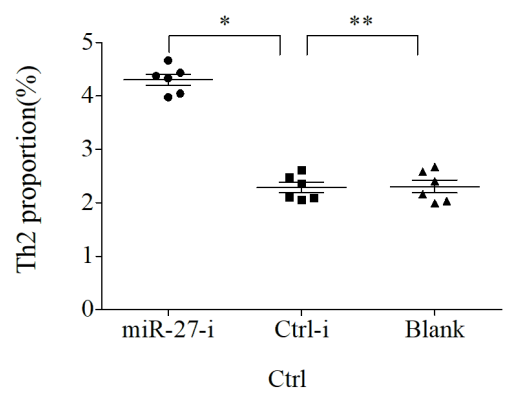

K .

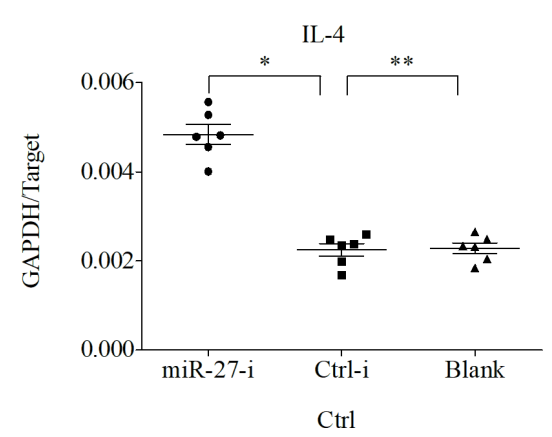

H.

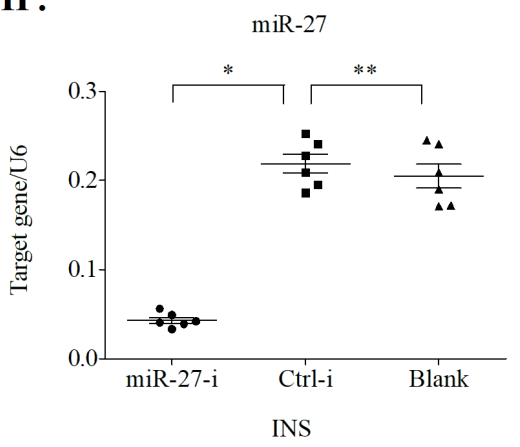

J.

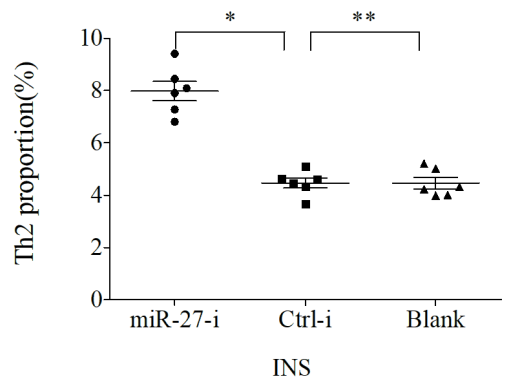

L .

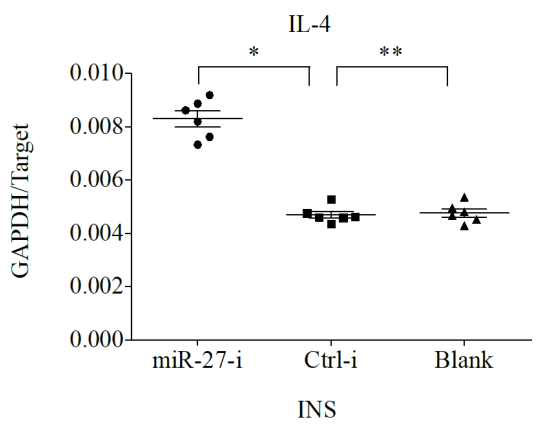

